\title{
Inventory from 1761 of the Deanery Church of Assumption of the Virgin Mary in Most
}

\author{
Jana Kratochvílová / 437764@mail.muni.cz
}

Department of Musicology, Masaryk University, Brno, CZ

\begin{abstract}
This study deals with the preserved inventory of The Church of the Assumption of the Virgin Mary in Most from 1761. It contains precious information about the musical production at those times. The inventory from 1761 and other inventories are confronted with preserved archival materials from State District Archives in Most. It is possible to trace changes in the instrumentation of the aforementioned church on the basis of preserved sources. Apart from the other inventories, the one from 1761 contains a special list of sheet music from the period of the Renaissance and Early Baroque. This list is presented in a form of an edition.
\end{abstract}

\section{Keywords}

inventory, Most, deanery church, musical instruments, musical manuscripts, sheet music

The article came into being as part of the project "Hudební inventáre raného novověku v českých zemích" supported by the Grantová agentura České republiky (project no. GA16-17615S). 


\section{Introduction}

From the point of musical history, few people have been paying attention to the royal town Most, even though the deanery Church in Most has played an important role in local cultural history. Preserved archival and museum sources provide evidence of rich musical production, which was concentrated not only in the deanery church but also in other sacral buildings in Most. The study then separately focuses on the inventory from 1761, which, apart from the repertoire from the times when the inventory was made, includes a unique list of sheet music mostly from the era of the High Renaissance.

\section{The Deanery Church}

The town of Most has gone through several tough tests during its cultural evolution. At the turn of the $15^{\text {th }}$ and $16^{\text {th }}$ centuries, the city experienced an economic growth - development of several means of businesses led to Most becoming the centre of crafts and trades, and the city gaining lands far from its borders. On Sunday, $11^{\text {th }}$ of March, 1515 Most was hit by an enormous fire with fatal consequences. The original deanery church, Early Gothic three-nave basilica built in the years 1253-1257, burnt down completely. ${ }^{1}$ That is why it was decided to build a new one. Most was responsible for the new building for it had the right of patronage over the deanery church. This also represented a considerable financial burden. Immediately after the fire, $25^{\text {th }}$ of May 1515 , the cardinal and the papal legate Tomáš allowed a money collection to build the new church. The king Vladislav Jagellonský (Vladislaus II of Hungary) as well as the Polish king Zikmund I (Sigismund I. the Old) showed their support for the city when, thanks to their initiative, the pope Leo X had issued a bull that granted indulgence to everyone in Bohemia, Moravia, Silesia and Lusatia who would donate to the building of the deanery church as much as they would need for a week or provided materials and other help for the construction. Next year, 1516, Ludvík Jagellonský (Louis II of Hungary) also supported the construction by allowing burghers another money collection. ${ }^{2}$ The cornerstone of the new deanery church of The Church of the Assumption of the Virgin Mary was laid on the $15^{\text {th }}$ of August 1517. The renovation was entrusted to the architect Jakub Heilmann of Schweinfurt. A lot of masons, masters and parlers took part in the slow construction of the new church, but despite that Heilmann's plans were followed. Eventually, in 1597, the deanery church was finished and consecrated by Prague archbishop Zbyněk Berka of Dubá. ${ }^{3}$ The inventories included in this study document a rich musical production of the

1 MANNLOVÁ, Heide. Kulturni památka Most: Děkanský kostel a jeho stavitelé. Praha: Pro odbor kultury a Okresní muzeum v Mostě k tisku připravila Propagační tvorba, 1989, p. 9-17.

2 MYŠIČKA, Martin. Kniha účtů ke sbírce na stavbu děkanského kostela. In 500 let kostela Nanebevzeti Panny Marie v Mostě. V Mostě: Oblastní muzeum, 2018, p. 7.

3 FÖRSTER, Jürgen. Stavitel Jacob Haylmann ze Schweinfurtu, město Annaberg a jejich vliv na vybudování 
deanery church. Thanks to the unique collections of preserved sheet music and musical instruments it is possible to further compare the information.

\section{The Corporis Christi Confraternity}

Archival sources and preserved collections of musical instruments and sheet music are evidence of rich musical events in the royal city. Even though most of the musical production was developing by the deanery church, the Minorites took part, too. ${ }^{4}$ While studying the musical history in Most another question appears - the existence of literary a brotherhood. Karel Konrád talks about the existence of a latin confraternity of literary brotherhood in Dějiny posvátného zpěvu staročeského, which was established in Most after Protestantism had widely spread during the $16^{\text {th }}$ century, ${ }^{5}$ but this is rather author's own hypothesis. The confraternity is not mentioned under this name in other sources that have been examined already. However, the confraternity Corporis Christi demonstrably existed in Most. Its activity is documented from 1411 up to the $18^{\text {th }}$ century and it survived even the era of the Hussite Wars. ${ }^{6}$ In 1415 the brotherhood founded a chaplain benefice by the parish church. Its holder was obliged to serve a mass three times a week and on various feasts. ${ }^{7}$ The foundation of the confraternity was mentioned in the book Geschichte der königlichen Stadt Brüx. ${ }^{8}$ Based on a note in the preserved inventory from 1727 it is evident that the confraternity of God's Body obtained a small organ for the deanery church. ${ }^{9}$ We know about the musical activities from the contract with an auxiliary singer Sukowanský in 1697. In the requirements it is stated that the adjuvant had to be present for singing during regular church rituals as well as the confraternity of Corporis Christi ones. ${ }^{10}$ The position of adjuvant or also adstant was much more complex than just auxiliary. They were musically capable individuals, who were not only singers, but also played an instrument, as it is observable from other contracts of individual

děkanského chrámu v Mostě. In 500 let kostela Nanebevzeti Panny Marie v Mostě. V Mostě: Oblastní muzeum, 2018, p. 61.

4 VLČEK, Pavel, Dušan FOLTÝN a Petr SOMMER. Encyklopedie českých klášterů. Praha: Libri, 1997, p. 379-382.

5 KONRÁD, Karel. Dějiny posvátného zpěvu staročeského. Díl 2., č. 1., od XV. věku do zrušení literátskych bratrstev, Věk a dějiny literárních bratrstev. Praha: Cyrillo-Methodějská kněhtiskárna, 1883, p. 138.

6 PÁTKOVÁ, Hana. Bratrstvie ke cti Božie: poznámky ke kultovni činnosti bratrstev a cechů ve středověkých Čechách. Praha: KLP, 2000, p. 17.

7 PÁTKOVÁ, Hana. Bratrstvie ke cti Božie: poznámky ke kultovni činnosti bratrstev a cechů ve středověkých Čechách. Praha: KLP, 2000, p. 17.

8 CORI, Johann Nepomuk, Ferdinand von HOCHSTETTER a Franz SIEGEL. Geschichte der königl. Stadt Brüx bis zum Jahre 1788. Brüx: Im Verlage der Stadt Brüx, 1889, p. 212.

$9 \quad[. .$.$] de andere kleine Orgel ist brauchbar und von der löblich: Bruderschaft SS: Corp: Christ: allhier Verschaft. State$ Archiv Prague, Archive of the Prague Archdioecesis (APA), box nr. 2395.

10 State regional archive Most, Archive of the City of Most (1315-1945), Inv. nr. 3438 (auxiliary musicians in the deanery church 1677-1803). 
adjuvants. The first preserved contract is from 1677 for an adjuvant called Dominic Henners. ${ }^{11}$ This contract also includes instructions which the adjuvant should abide. His duties were singing the choral during various church ceremonies, and he was supposed to be capable of participating on the production of figural music. With organ accompaniment Henners was obliged to sing during masses, processions or vespers. Apart from already mentioned duties he was entrusted with education and gathering sheet music for the choir. ${ }^{12}$ Similar requirements are included in other preserved contracts with adjuvant such as Scabin Sukowanský, Johann Benedict Müller or Maxmilián Urban Loth. ${ }^{13}$

\section{The Inventories of the Deanery Church}

The inventories of the deanery church of the Assumption of the Virgin Mary in Most are a unique evidence of local musical activities. These lists come mainly from the second half of the $18^{\text {th }}$ century and they are preserved in the National Archives in Prague. ${ }^{14}$ Other inventories are located in the diocesan archive in Litoměřice. Apart from those, other inventories of the deanery church were found in the State District Archives in Most. Those inventories come from the years 1777, 1793, 1824 and 1846. ${ }^{15}$ Apart from 1793 , there is information about sheet music and musical instruments in all of the abovementioned inventories from Most. The inventory from 1777 is preserved in two almost identical copies which were put together by the same authors, Adalbert Karel Kölbel and Johann Wenzel Petz, on the same date of $24^{\text {th }}$ of January. The changes in the instrumentation are visible just from the inventories from 1824 and 1846 . Said inventories of the deanery church, which include the list of musical instruments also mention their quantity. Thus, we can track gradual changes in the instrumentation.

Next valuable sources, which complete the notes in the inventories, are collections of musical instruments and sheet music from the Regional Museum in Most. This sheet music represents a significant source for the study of musical practice in Most at that time and comes mainly from the $18^{\text {th }}$ century. A considerable amount of musical manuscripts comes from the natives of Most - Andreas Hammerschmidt (c1611-1675), Anton Laube (1718-1784) or Florian Leopold Gassmann (1729-1774). For better clarity in the above-mentioned inventories and its musical contents a chart is provided below:

\footnotetext{
11 Ibid.

12 ČERNÝ, Milan. Chrámoví hudebníci a hudebně liturgická praxe při duchcovském kostele zvěstování Panny Marie v první polovině 18. století. In. Porta Bohemica: sborník historických prací, č.8. Ústí nad Labem: Albis international, 2001, p. 100.

13 State regional archive Most, Archive of the City of Most (1315-1945), Inv. nr. 3438 (auxiliary musicians in the deanery church 1677-1803).

14 State Archiv Prague, Archive of the Prague Archdioecesis (APA), box nr. 2395.

15 State regional archive Most, Archive of the City of Most (1315-1945), Inv. nr. 2752 (inventories of the deanery church).
} 


\begin{tabular}{|l|c|c|}
\hline Inventory & Sheet music & Musical instruments \\
\hline 1724 & $\times$ & $\times$ \\
\hline 1727 & $\times$ & $\cdot$ \\
\hline 1761 & $\cdot$ & $\cdot$ \\
\hline 1768 & $\cdot$ & $\cdot$ \\
\hline 1777 & $\cdot$ & $\cdot$ \\
\hline 1793 & $\times$ & $\times$ \\
\hline 1824 & $\cdot$ & $\cdot$ \\
\hline 1846 & $\cdot$ & $\cdot$ \\
\hline
\end{tabular}

Tab. 1 Presence of sheet music and musical instruments in inventories in Most.

\section{The Organs and Instrumentation of the Deanery Church}

The organ is an interesting object of study. The first mention of the organs is in the inventory from 1727 and it talks about "a big organ, to this date unusable". ${ }^{16}$ It was probably the organ for which the contract was issued on $23^{\text {rd }}$ of January 1611, constructed by David Decker, ${ }^{17}$ a Thuringian organ builder operating in Bohemia. ${ }^{18}$ However, the contract mentions only a renovation of the existing instrument. ${ }^{19}$ The organ was probably originally constructed in 1585 by Gabriel Raffael Rodensteen. ${ }^{20}$ The instrument was then rebuilt during years $1675-1678$ by Michael Franz Kannhauser. ${ }^{21}$ The inventory also mentions a small, usable organ, which was bought for the deanery church by the Corporis Christi Confraternity. In the place of the above-mentioned unusable organ a construction of a new one with 23 registers, began in 1739. ${ }^{22}$ The contract for the construction issued the rector and the Corporis Christi Confraternity with Wenzel Starck, who then cooperated with Jan Adam Pleyer. ${ }^{23}$ The new organ had later been repaired several times as it is evident from the preserved contracts. According to the documents it is obvious

16 Ein große Orgel, zu dato unbrauchbahr. State Archiv Prague, Archive of the Prague Archdioecesis (APA), box nr. 2395.

17 CORI, Johann Nepomuk, Ferdinand von HOCHSTETTER a Franz SIEGEL, op. cit., p. 213.

18 HORÁK, Tomáš. Varhany a varhanárí Lounska, Žatecka a Podbořanska. Ústí nad Labem: Albis international, 2003, p. 73.

19 State regional archive Most, Archive of the City of Most (1315-1945), Inv. nr. 2913 (contract between the town of Most and the organ builder David Decker from Zeilenrode in Voigtland).

20 KOCOUREK, Jiří. Orgelland Böhmen. In Ars Organi 57. Mettlach. 2009, č. 1, p. 5-18.

21 CORI, Johann Nepomuk, Ferdinand von HOCHSTETTER a Franz SIEGEL, op. cit., p. 306.

22 HORÁK, Tomáš. Varhany a varhanáři Ústecka. Ústí nad Labem: Město Ústí nad Labem, 2002, p. 71-73.

23 State regional archive Most, Archive of the City of Most (1315-1945), Inv. nr. 2915 (contract between the town of Most and the organ builder Wenzel Starcke from Loket / Ellbogen). 
that Wenzel Starck did not only participate in the construction, but he also renovated the organ on the small choir. ${ }^{24}$

The inventory from 1727 contains, apart from the organ and one positive organ, only three violins and one viola. Overall, the inventory from 1761 lists forty instruments, including the unusable ones. Apart from the instruments themselves the inventory also mentions accessories such as mouthpieces, bows or timpani sticks. If we compare preserved inventories with each other, we can trace even the condition of listed instruments. In the following inventory from 1768 there is a significant deterioration of the musical instruments since the year 1761. For example, a violoncello firstly mentioned in the inventory from 1761 is listed as "very bad" in the following inventory. The same goes for a viola of which there is one piece firstly mentioned in 1727. In the inventory from 1761 two violas are present, one of which is listed as "unusable". Then in 1777 just one viola is mentioned, however unusable. The way cornetts are listed in 1761 raises some doubts as it is written in plural and suggests existence of multiple instruments. However, the number states only one item. In 1768, 1824, 1846 and 1878 the instrument is written in singular, therefore we can assume that just one exemplar was present in the instrumentation, even though in 1777 the same inaccuracy occurs again. Newly two clarinets are mentioned in 1768. In the case of trumpets, there is a significant loss - in 1761 the inventory states four new, four old and four unusable trumpets. The next inventory from 1768 states just six trumpets and in 1777 just four trumpets are left and another six are listed as old and unusable. Identification of specific instruments across preserved inventories is difficult, due to the fact that not all instruments or their condition are mentioned in every inventory. In cases where the number of certain instrument is present and remains the same across the inventories, the identification is much easier, and the instruments are probably the same in all cases. The inventory from 1777 also includes a fairly detailed list of 39 musical instruments. There is also a separate a list of accessories such as bows, drumsticks etc. Another part provides a list of old and unusable instruments, 16 of them in total and half of a bassoon.

\begin{tabular}{|c|c|c|c|c|c|c|}
\hline $\begin{array}{l}\text { Inventory } \\
1727\end{array}$ & \begin{tabular}{|l|} 
Inventory \\
1761
\end{tabular} & \begin{tabular}{|l|} 
Inventory \\
1768 \\
\end{tabular} & \begin{tabular}{|l} 
Inventory \\
1777
\end{tabular} & \begin{tabular}{|l|} 
Inventory \\
1824 \\
\end{tabular} & \begin{tabular}{|l|} 
Inventory \\
1846 \\
\end{tabular} & $\begin{array}{l}\text { Inventory } \\
1878\end{array}$ \\
\hline $\begin{array}{l}1 \text { Orgel auf } \\
\text { den kleinen } \\
\text { Chor 4: Fuß }\end{array}$ & & 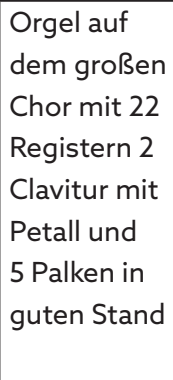 & & \begin{tabular}{|l} 
Orgel auch \\
dem großen \\
Chorn mit 22 \\
Registern, \\
2 Claviature \\
mit Pedalen \\
und 5 bölgen \\
in guten \\
Stande in ---
\end{tabular} & & \\
\hline
\end{tabular}

24 Ibid. 


\begin{tabular}{|c|c|c|c|c|c|c|}
\hline $\begin{array}{l}\text { Inventory } \\
1727\end{array}$ & \begin{tabular}{|l|} 
Inventory \\
1761
\end{tabular} & $\begin{array}{l}\text { Inventory } \\
1768\end{array}$ & \begin{tabular}{|l|} 
Inventory \\
1777
\end{tabular} & \begin{tabular}{|l|} 
Inventory \\
1824 \\
\end{tabular} & \begin{tabular}{|l|} 
Inventory \\
1846
\end{tabular} & \begin{tabular}{|l|} 
Inventory \\
1878 \\
\end{tabular} \\
\hline \multirow[t]{3}{*}{$\begin{array}{l}1 \text { kleines } \\
\text { positiv Zum } \\
\text { herumbtra- } \\
\text { gen }\end{array}$} & & $\begin{array}{l}\text { kleinere Orgl } \\
\text { auf dem klei- } \\
\text { nen Chore } \\
\text { mit } 10 \text { Regis- } \\
\text { tern Petall } \\
\text { und } 3 \text { Pälken } \\
2 \text { Clavitum }\end{array}$ & & $\begin{array}{l}\text { Orgel kleiner } \\
\text { auf dem } \\
\text { kleinen } \\
\text { Chorn mit } 10 \\
\text { Registern, } \\
\text { Pedalen, } 2 \\
\text { Claviaturn? } \\
\text { und } 3 \text { bölgen } \\
\text { gut }\end{array}$ & & \\
\hline & 2 Violon & $\begin{array}{l}\text { zwey Violo- } \\
\text { ne woran } 1 \\
\text { unbauchbar }\end{array}$ & 2 Violone & $\begin{array}{l}\text { Violone } \\
\text { zwey Stuck, } \\
\text { ? eineß un- } \\
\text { brauchbar }\end{array}$ & & $\begin{array}{l}\text { Violons } \\
\text { oder Con- } \\
\text { trabässe } \\
\text { sammt } \\
\text { Bogen und } \\
\text { Spanner }\end{array}$ \\
\hline & 1 ViolonZello & $\begin{array}{l}\text { ein violon- } \\
\text { zelle sehr } \\
\text { schlecht }\end{array}$ & 1 Violonzello & $\begin{array}{l}\text { Ein Violon- } \\
\text { celle schle- } \\
\text { cht }\end{array}$ & $\begin{array}{l}1 \text { altes un- } \\
\text { brauchbare } \\
\text { Violonzell }\end{array}$ & $\begin{array}{l}1 \text { altes Vio- } \\
\text { loncžell }\end{array}$ \\
\hline \multirow[t]{6}{*}{$\begin{array}{l}3 \text { kleinen } \\
\text { Geigen }\end{array}$} & 4 violinen & $\begin{array}{l}\text { Violine } 2 \\
\text { gute samt } \\
\text { zugehör in } \\
\text { Futrale }\end{array}$ & & \begin{tabular}{|l|} 
Violine zwey \\
gute sammt \\
zugehör in \\
futteral
\end{tabular} & $\begin{array}{l}8 \text { Violinen } \\
\text { samt bögen } \\
\text { et. futral }\end{array}$ & $\begin{array}{l}8 \text { Violinen } \\
\text { samt Bögen } \\
\text { und futreral }\end{array}$ \\
\hline & & $\begin{array}{l}\text { d. } 5 \text { schle- } \\
\text { chte samt } \\
\text { Bögen }\end{array}$ & $\begin{array}{l}5 \text { Violinen, } \\
\text { welche } \\
\text { schlecht sind }\end{array}$ & $\begin{array}{l}\text { fünf schle- } \\
\text { chte sammt } \\
\text { bögen }\end{array}$ & & \\
\hline & $\begin{array}{l}1 \text { geigen mit } \\
\text { einem Futtral }\end{array}$ & & $\begin{array}{l}3 \text { deto von } \\
\text { verschiede- } \\
\text { nen Gutthä- } \\
\text { tern neue }\end{array}$ & & & \\
\hline & & & $\begin{array}{l}1 \text { deto von } \\
\text { /:Tit:/ Herrn } \\
\text { Petz }\end{array}$ & & & \\
\hline & $\begin{array}{l}\text { dann zwey } \\
\text { buxbaumene } \\
\text { Flautten von } \\
\text { /: titl:/ Seel: } \\
\text { H Dechant } \\
\text { auf hießiges } \\
\text { Chor=Ver- } \\
\text { schenckt } \\
\text { werden }\end{array}$ & & 2 Flauten & & & 1 stk flöte \\
\hline & & ein altViola & & $\begin{array}{l}\text { Eine Alto- } \\
\text { viola }\end{array}$ & $\begin{array}{l}1 \text { alte Viola } \\
\text { samt bogen }\end{array}$ & $\begin{array}{l}1 \text { alte Viola } \\
\text { sammt Bogen }\end{array}$ \\
\hline
\end{tabular}


Inventory from 1761 of the Deanery Church of Assumption of the Virgin Mary in Most

\begin{tabular}{|c|c|c|c|c|c|c|}
\hline $\begin{array}{l}\text { Inventory } \\
1727\end{array}$ & \begin{tabular}{|l|} 
Inventory \\
1761
\end{tabular} & \begin{tabular}{|l|} 
Inventory \\
1768 \\
\end{tabular} & \begin{tabular}{|l|} 
Inventory \\
1777 \\
\end{tabular} & \begin{tabular}{|l|} 
Inventory \\
1824 \\
\end{tabular} & \begin{tabular}{|l|} 
Inventory \\
1846 \\
\end{tabular} & \begin{tabular}{|l|} 
Inventory \\
1878 \\
\end{tabular} \\
\hline \multirow[t]{12}{*}{1 Pratschen } & $\begin{array}{l}1 \text { Pratschen } \\
\text { so brauchbar }\end{array}$ & & $\begin{array}{l}1 \text { Bratz schier } \\
\text { unbrauchbar }\end{array}$ & & & \\
\hline & $\begin{array}{l}\text { Item eine un- } \\
\text { brauchbare }\end{array}$ & & & & & \\
\hline & 2 Huboes & $\begin{array}{l}\text { Houtboi } 1 \\
\text { Stuck gut } \\
\text { mit silbern } \\
\text { klappen }\end{array}$ & 2 Huboes & \begin{tabular}{|l|} 
Oboe ein \\
Stück gut mit \\
silb neüen \\
klappen
\end{tabular} & $\begin{array}{l}1 \text { gute Oboe } \\
\text { mit } 4 \text { Mittel- } \\
\text { stücken von } \\
\text { buxbaum } \\
\text { und } 2 \text { silber- } \\
\text { nen Klappen }\end{array}$ & $\begin{array}{l}1 \text { Oboe mit } \\
4 \text { Mittel- } \\
\text { stücken von } \\
\text { Buxbaum et } \\
2 \text { silbernen } \\
\text { Klappen }\end{array}$ \\
\hline & & & & & \begin{tabular}{|l|}
1 ältere \\
Oboe von \\
ordinären \\
harten holz \\
\end{tabular} & \begin{tabular}{|l|}
1 ältere \\
Oboe von \\
ordinären \\
harten holz
\end{tabular} \\
\hline & \begin{tabular}{|l}
4 kurtze $D$ \\
Trompeten \\
so unbrauch- \\
bar
\end{tabular} & $\begin{array}{l}\text { Trompeten } 4 \\
\text { Stuck in D: }\end{array}$ & 4 Trompeten & \begin{tabular}{|l|} 
Trompeten \\
vier Stück \\
in d. mit 4 \\
krummbögen \\
2 gut und 4 \\
mittelmäßig
\end{tabular} & $\begin{array}{l}4 \text { alte Trom- } \\
\text { peten sammt } \\
6 \text { Krumbö- } \\
\text { gen }\end{array}$ & $\begin{array}{l}4 \text { alte Trom- } \\
\text { peten sammt } \\
6 \text { Krumbö- } \\
\text { gen }\end{array}$ \\
\hline & $\begin{array}{l}4 \text { alte Trom- } \\
\text { peten }\end{array}$ & 1 Stuck in a. & & $\begin{array}{l}\text { ein Stück in } \\
a / c\end{array}$ & & $\begin{array}{l}2 \text { Trompeten } \\
\text { mit Aufzug }\end{array}$ \\
\hline & \begin{tabular}{|l}
4 Neue \\
Trompeten
\end{tabular} & 1 Stuck in $\mathrm{g}$. & & $\begin{array}{l}\text { ein Stück } \\
\text { in g. }\end{array}$ & & \\
\hline & & & $\begin{array}{l}1 \text { Trompet } \\
\text { beym Stadt } \\
\text { Thurmer }\end{array}$ & $\begin{array}{l}\text { Eine Trom- } \\
\text { pete beim } \\
\text { Thurner }\end{array}$ & & \\
\hline & & $\begin{array}{l}\text { ein fagot } \\
\text { unbrauchbar }\end{array}$ & 1 Fagott & $\begin{array}{l}\text { Ein Fagot } \\
\text { unbrauchbar }\end{array}$ & \begin{tabular}{|l|}
1 alter fagot \\
mit 6 mes- \\
singenen \\
Klappen et \\
einem Es \\
\end{tabular} & \begin{tabular}{|l}
1 alte Fagot \\
mit 6 mes- \\
singenen \\
Klappen und \\
einem Es \\
\end{tabular} \\
\hline & $\begin{array}{l}2 \text { alte Wald- } \\
\text { thorn }\end{array}$ & & $\begin{array}{l}1 \text { paar Neue } \\
\text { Wald Hörner }\end{array}$ & $\begin{array}{l}\text { Ein paar alte } \\
\text { Waldhörnen } \\
\text { mit ? und } \\
\text { krummbögen }\end{array}$ & & $\begin{array}{l}2 \text { Waldhör- } \\
\text { ner }\end{array}$ \\
\hline & 2 Waldhorn & & $\begin{array}{l}2 \text { Alte } \\
\text { deto doch } \\
\text { brauchbahr }\end{array}$ & $\begin{array}{l}\text { Ein paar alte } \\
\text { neue }\end{array}$ & & \\
\hline & 3 Possaunen & \begin{tabular}{|l|} 
possaunen \\
zwey stuck \\
mittelmäsig, 1 \\
sehr schlecht \\
\end{tabular} & 3 Posaunen & \begin{tabular}{|l} 
Posaunen \\
zwey Stück \\
mittelmäsig \\
1 ? schlecht
\end{tabular} & \begin{tabular}{|l}
3 alte Po- \\
saunen mit \\
2 Mundstüc- \\
ken
\end{tabular} & \begin{tabular}{|l}
3 alte Po- \\
saunen mit \\
2 Mundstüc- \\
ken
\end{tabular} \\
\hline
\end{tabular}


Inventory from 1761 of the Deanery Church of Assumption of the Virgin Mary in Most

\begin{tabular}{|c|c|c|c|c|c|c|}
\hline $\begin{array}{l}\text { Inventory } \\
1727\end{array}$ & \begin{tabular}{|l|} 
Inventory \\
1761
\end{tabular} & $\begin{array}{l}\text { Inventory } \\
1768\end{array}$ & $\begin{array}{l}\text { Inventory } \\
1777\end{array}$ & \begin{tabular}{|l|} 
Inventory \\
1824
\end{tabular} & \begin{tabular}{|l|} 
Inventory \\
1846
\end{tabular} & $\begin{array}{l}\text { Inventory } \\
1878\end{array}$ \\
\hline & & & & & & $\begin{array}{l}1 \text { neue Pos- } \\
\text { saune }\end{array}$ \\
\hline & $\begin{array}{l}\text { alte Posau- } \\
\text { nen }\end{array}$ & & & & & \\
\hline & $\begin{array}{l}1 \text { baar Neue } \\
\text { Paucken } \\
\text { nebst zu- } \\
\text { gehörige } \\
\text { Spanner }\end{array}$ & & $\begin{array}{l}2 \text { Paucken } \\
1 \text { paar gutt, } \\
\text { und } 1 \text { paar } \\
\text { älter }\end{array}$ & & \begin{tabular}{|l|}
2 paar \\
Kupferne \\
Kesselpau- \\
ken mit 1 \\
Spanner, 2 \\
paar Schlägel \\
et 2 Riemen \\
\end{tabular} & $\begin{array}{l}2 \text { paar } \\
\text { Kupferne } \\
\text { Kesselpau- } \\
\text { ken mit } 1 \\
\text { Spanner, } 2 \\
\text { paar Schlägel } \\
\text { et } 2 \text { Riemen }\end{array}$ \\
\hline & $\begin{array}{l}1 \text { baar alte } \\
\text { Paucken } \\
\text { nebst zu- } \\
\text { gehörige } \\
\text { Spanner }\end{array}$ & & & & & \\
\hline & $\begin{array}{l}1 \text { baar Pau- } \\
\text { ken? }\end{array}$ & & & $\begin{array}{l}\text { dreyzwey } \\
\text { Paar Pauc- } \\
\text { ken sammt } \\
\text { Schlägeln } \\
\text { und einem } \\
\text { Spanner }\end{array}$ & & \\
\hline & $\begin{array}{l}1 \text { baar alte } \\
\text { Kirchen } \\
\text { adjuvanten } \\
\text { Paucken } \\
\end{array}$ & & & & & \\
\hline & 1 Cincken & 1 Zinke & $\begin{array}{l}1 \text { Zinckten } \\
\text { beym Stadt } \\
\text { Thurmer }\end{array}$ & \begin{tabular}{|l} 
Eine zinke \\
sammt vor- \\
mund Stück \\
von Leden
\end{tabular} & $\begin{array}{l}1 \text { Zinke von } \\
\text { holtspänen } \\
\text { mit leder } \\
\text { überzogen } \\
\end{array}$ & $\begin{array}{l}1 \text { Zinke von } \\
\text { holtspänen } \\
\text { mit leder } \\
\text { überzogen }\end{array}$ \\
\hline & & $\begin{array}{l}\text { zwey stuck } \\
\text { Clarinetten } \\
\text { samt zwey }\end{array}$ & & $\begin{array}{l}\text { Clarinete } \\
\text { zwey Stück } \\
\text { sammt zwey } \\
\text { Satzeln und } \\
\text { zwey Mittel } \\
\text { stücken }\end{array}$ & & $\begin{array}{l}2 \text { stk neue } A \\
\text { Clarinetten }\end{array}$ \\
\hline & & & & & & $\begin{array}{l}2 \text { stk neue } \mathrm{C} \\
\text { Clarinetten }\end{array}$ \\
\hline & & $\begin{array}{l}\text { zwey stuck } \\
\text { violin futral }\end{array}$ & & $\begin{array}{l}\text { drey Stück } \\
\text { alte Violin } \\
\text { futterale }\end{array}$ & & \\
\hline
\end{tabular}


Inventory from 1761 of the Deanery Church of Assumption of the Virgin Mary in Most

\begin{tabular}{|c|c|c|c|c|c|c|}
\hline \multirow[t]{15}{*}{\begin{tabular}{|l|} 
Inventory \\
1727 \\
\end{tabular}} & \begin{tabular}{|l|} 
Inventory \\
1761 \\
\end{tabular} & $\begin{array}{l}\text { Inventory } \\
1768\end{array}$ & $\begin{array}{l}\text { Inventory } \\
1777 \\
\end{array}$ & \begin{tabular}{|l|} 
Inventory \\
1824 \\
\end{tabular} & $\begin{array}{l}\text { Inventory } \\
1846\end{array}$ & $\begin{array}{l}\text { Inventory } \\
1878\end{array}$ \\
\hline & & $\begin{array}{l}\text { ein Violon } \\
\text { spanner }\end{array}$ & $\begin{array}{l}1 \text { Violon } \\
\text { Spanner }\end{array}$ & $\begin{array}{l}\text { Ein Violons- } \\
\text { panner von } \\
\text { holz }\end{array}$ & & \\
\hline & $\begin{array}{l}10 \text { Krumppö- } \\
\text { gen }\end{array}$ & & $\begin{array}{l}12 \text { Große } \\
\text { und kleine } \\
\text { Bögen wo- } \\
\text { runter } 3 \text { un- } \\
\text { brauchbahr }\end{array}$ & & & \\
\hline & 6 auffsteln & & & & & \\
\hline & $\begin{array}{l}4 \text { Sardinl zu } \\
\text { denen Trom- } \\
\text { peten }\end{array}$ & & & & $\begin{array}{l}3 \text { hölzerne } \\
\text { Trompeten } \\
\text { Sardinen } \\
\end{array}$ & $\begin{array}{l}3 \text { hölzerne } \\
\text { Trompeten } \\
\text { Sardinen } \\
\end{array}$ \\
\hline & \begin{tabular}{|l}
2 Mund \\
stuck zu de- \\
nen Neuen \\
Waldthorn \\
\end{tabular} & & $\begin{array}{l}1 \text { Mund } \\
\text { Stuckle zum } \\
\text { Waldhorn }\end{array}$ & & & \\
\hline & & & $\begin{array}{l}1 \text { kleine } \\
\text { Krumbbögen } \\
\text { zu dem Wal- } \\
\text { dhornen }\end{array}$ & & & \\
\hline & & & $\begin{array}{l}1 \text { Größere } \\
\text { deto }\end{array}$ & & & \\
\hline & & & $\begin{array}{l}2 \text { d: sten } \\
\text { zum Wald- } \\
\text { hornen }\end{array}$ & & & \\
\hline & & & $\begin{array}{l}1 \text { deto zum } \\
\text { Trompeten }\end{array}$ & & & $\begin{array}{l}\text { Mehrere El- } \\
\text { len Schnüre } \\
\text { und Quasten } \\
\text { zu den Trom- } \\
\text { peten }\end{array}$ \\
\hline & & & $\begin{array}{l}1 \text { Spanner } \\
\text { hierzu }\end{array}$ & & & \\
\hline & & & $\begin{array}{l}2 \text { Schlägln zu } \\
\text { dem Pauc- } \\
\text { ken }\end{array}$ & & & \\
\hline & & & $\begin{array}{l}\text { Alte un- } \\
\text { brauchbahre } \\
\text { Instumenta: }\end{array}$ & & & \\
\hline & & & $\begin{array}{l}1 \text { Alter viel- } \\
\text { leicht } 100 \\
\text { Jähriger } \\
\text { Violon }\end{array}$ & & & \\
\hline & & & 2 Violinen & & & \\
\hline
\end{tabular}




\begin{tabular}{|l|l|l|l|l|l|l|}
\hline $\begin{array}{l}\text { Inventory } \\
\mathbf{1 7 2 7}\end{array}$ & $\begin{array}{l}\text { Inventory } \\
\mathbf{1 7 6 1}\end{array}$ & $\begin{array}{l}\text { Inventory } \\
\mathbf{1 7 6 8}\end{array}$ & $\begin{array}{l}\text { Inventory } \\
\mathbf{1 7 7 7}\end{array}$ & $\begin{array}{l}\text { Inventory } \\
\mathbf{1 8 2 4}\end{array}$ & $\begin{array}{l}\text { Inventory } \\
\mathbf{1 8 4 6}\end{array}$ & $\begin{array}{l}\text { Inventory } \\
\mathbf{1 8 7 8}\end{array}$ \\
\hline & & & 3 Translatum & & & \\
\hline & & & 1 Bratz & & & \\
\hline & & & 6 Trompeten & & & \\
\hline & & & 3 Bassaunen & & & \\
\hline & & & 2 alte Bögen & & & \\
\hline & & $\begin{array}{l}\text { 1 paar alte } \\
\text { Paucken }\end{array}$ & & & \\
\hline & & $\begin{array}{l}1 \text { altes In- } \\
\text { strument } \\
\text { gleich einen } \\
\text { halben Fa- } \\
\text { got, deren } \\
\text { kein Nahme } \\
\text { zu geben ist }\end{array}$ & & & \\
\end{tabular}

Tab. 2 The changes in the instrumentation of the deanery church in Most.

\section{The inventory from 1761}

The inventory from 1761, which is the subject of this study, includes relatively extensive information about the church's possessions. It was written on the date of $12^{\text {th }}$ of February by the new dean Joseph Böhm. Great attention was paid to metal objects (tin, copper, brass etc.). Paintings and statues located either in a sacristy or in a chapel are also listed in detail. There is a summary of financial means of the church at the very end of the inventory. The inventory also includes an important list of musical instruments and sheet music. This is preceded with a listing of liturgical books. First, just a short, listing of sheet music with the heading "Bey dem grossen Chor befinden sich musicalia. als" registres 12 items in total..$^{25}$ It includes four vespers, two litanies, hymn and four passion chants for the Holy Week. ${ }^{26}$

Bey dem grossen Chor befinden sich musicalia. $a l^{27}$

Vesperae seu psalmi per totum annum

Auth[ore] Jacobi 1 stuckh.

Vesperae de Dominica et B[eatae] V[irginis] Mariae

Author[e] Thalman[us] 1 stuck.

Vesperae de Dominica et S[ancti]S[simi] Apostolis Auth[ore]

25 There is probably 12 items. In the hymn entry there is not an exact number, in the note Stuckh quantity is not provided at all.

26 Bound in one volume.

27 All inventories are transcribed completely, including stylistical errors and original properties of the text. 
Philippi, 1 stuck.

Vesperae de Dominica breviores Auth[ore] Reickweih (!), 1 stuckh.

Johann Georg Reichwein (1640-1691): Vesperae Dominicales breves à 4 Voc: Violini 2. Tromboni 2 ad libitum con Organo e Violone Authore Joanna Georgio Reichwein.

Lytaniae de S[ancta] Anna . 2 stuckh.

Lytaniae de S[ancti]S[sime] Nomine Jesu. 1 stuckh

Hymnus Israel es tu pro Dominica Palmarum . . stuckh

Quatuor passiones pro hebdomada Sancta zusammen in einen bundt.

Nebst dießen befinden sich nach verschiedene andere Musicalia so aber Theils sehr veraltet, Theils auch in denen Stimmen nicht vergäntzet.

The list of sheet music includes precious information not only about its liturgical purpose, but also about the authorship of the compositions. Composers, who are listed in the inventory from 1761, are Jacobbi, Thalman, Philippi and Reickweih. Most likely they are composers Václav Jan Antonín Jacob (1685-1734) and Franz Daniel von Thallmann (1668-1740). Next author is probably Jan Jiří Philippi (c1670-1722). ${ }^{28}$ The name Reickweih is written erroneously, correctly it is a composer Johann Georg Reichwein (1640-1691). Thanks to the detailed entry it is possible to identify the compositions in RISM. Another information about the sheet music is also provided by other preserved inventories. Some of the sheet music is listed even with its author. In the inventory from 1777 they are listed in a section called "An verschiedenen Musicalien". Mentioned pieces of sheet music were kept on the big choir and thus they are identical to those from the inventory from 1761, which had also been kept in the same place. Apart from the identical compositions in the inventory from 1777 there are also new names, while the above-mentioned composers are not present any more. As composers of said sheet music we can find here names such as Kayßer, Diez or Pögl. Under the last name Kayser a composer of church music Isfrid Kayser (1712-1771) is hiding. In the Regional Museum in Most there are two works of Kayser preserved - Vesperae cum consuetis Antiphonis de Beatissima Virgine ac Gloriosissima Dei Genetrice Maria, op. VII and Missae à 4. Vocibus ordinariis, op II. The note in the inventory „Opus sacrum in druck“ probably corresponds to the mentioned vespers. Other listed authors are most probably Peregrin Pögl (17111788) and Johann Sebastian Diez (1711-1793). Pögl's vespers as well as Opus sacrum by Johann Sebastian Diez are not preserved within the collections in Most.

After another list of books which were located under the small choir, in the inventory from 1761 also a list of musical instruments follows. For some of the instruments there is a note about their condition or their donator - e.g. the boxwood flutes were donated by local parish priest or one old timpani used to belong to local adjuvant whose role in the musical production is mentioned above. The list of musical instruments is followed by a list of sheet music with the heading "Eingebundene Musicalien in den Schrancken unter den Chor". It is a unique source, as there is not a similar list of compositions and their

28 STANĚK, Jan. Inventár̆ hudebnin piaristické koleje ve Slaném [online]. Brno, 2015 [online 2018-08-22]. Available from: https://is.muni.cz/th/y9fvj/. Bachelor thesis, Institute of Musicology, Faculty of Arts, Masaryk university. 
authors included in any other preserved inventory from Most. From the point of the repertoire the mentioned compositions come from the period of the High Renaissance and Early Baroque. The list includes works of composers such as Jacob Regnart, Orlando di Lasso, Jacobus Handl Gallus, Lodovico Viadana. However, some of the authors' names are garbled or completely changed e.g. Viadana whom Böhm writes as Diadana, Sebastian Ertel mentioned as Joanes, or Wilhelm Nordwig written as Nortwinck.

The inventory also presents some author's names which we cannot identify precisely. One of the first names we cannot accurately identify is the name Wussin. An author with this name is not present in any source, but the surname belongs to Daniel Wussin, who comes, as the eldest, from a family of engravers, publishers and booksellers of the $17^{\text {th }}$ and $18^{\text {th }}$ century. ${ }^{29}$ Hence a question arises, if there is a possibility that in the list there is a publisher mentioned instead of an author. Although according to available sources Daniel Wussin as a publisher concentrated more on professional law literature and used graphic. Wussin's firstborn son Jan František Wussin was a line engraver as well as his younger brother Kašpar Zachariáš Wussin. However, Daniel's younger son Kašpar continued as a publisher, too. ${ }^{30}$ So it seems unlikely that the names in the inventory would be the names of the typographer. Another, more complicated, is the name Joann Montani. Johann vom Berg (Joannes Montanus in Latin) was a typographer, a composer and a publisher from Nuremberg. In his and Ulrich Neuber's typography workshop in Nuremberg, they published Thesaurus musicus $1564 .{ }^{31}$ The entry in the inventory mentions only 2 pieces. This does not correspond to the whole published version, although it is possible that this work is not preserved complete. Another unidentifiable author is Theodorus Ring. Final note below the list points towards other, orderless and probably damaged, sheet music.

A diplomatic transcription of above-described list of sheet music from the inventory from 1761 follows. In case the author was identified his date of birth and death are provided. If found, data such as the year or place of publishing or publisher are listed after the title of a composition. For better clarity, a link to RISM is also present. For identification other musical lexicons were used. ${ }^{32}$ All errors in the text are marked with an exclamation mark.

29 VOIT, Petr. Encyklopedie knihy: starši knihtisk a přibuzné obory mezi polovinou 15. a počátkem 19. stoleti: papír, pismo a pismolijectvi, knihtisk a jiné grafické techniky, tiskaři, nakladatelé, knihkupci, ilustrátoři a kartografové, literárni typologie, textové a výtvarné prvky knihy, knižni vazba, knižni obchod. 2. vyd. Praha: Libri ve spolupráci s Královskou kanonií premonstrátů na Strahově, 2008, p. 1028.

30 Ibidem, p. 1029.

31 DANĚK, Petr. Historické tisky vokální polyfonie, rané monodie, hudební teorie a instrumentální hudby v českých zemích do roku 1630: se soupisem tiski̊ z let 1488-1628 uložených v Čechách. Praha: KLP, 2015, p. 69.

32 EITNER, Robert. Biographisch-Bibliographisches Quellen-Lexikon der Musiker und Musikgelehrten der christlichen Zeitrechnung bis zur Mitte des neunzehnten Jahrhunderts. Leipzig: Breitkopf und Härtel, 1900-1904. 
Eingebundene Musicalien in den Schrancken unter den Chor

1. Missae Sacrae Jacobi Regnardi 6 Theil in quarto

Jacob Regnart (c1540-1599): Missae sacrae ad imitationem selectissimarum cantionum suavissima harmonia, a quinque, sex, $\mathcal{E}$ octo vocibus. 1602 Frankfurt, Nikolaus Stein Wolfgang Richter, RISM R734.

2. Mottete, et Psalmi usq[ue] ad Sacelli (!) 7 Theil in quarto

Asprilio Pacelli (1570-1623): Motetae et psalmi, qui octonis vocibus concinuntur. 1607 Frankfurt, Nikolaus Stein (Wolfgang Richter) RISM P 25.

3. Introitus a Trinitate usq[ue] ad advent[um] 4 Theil in 4 to

This is a collection Choralis Constantinus by Heinrich Isaac (c 1450-1517).

[T:] PRIMVS TOMVS. | TENOR | CORALIS [sic!] CONSTANTINI, VT | vulgo vocant, opus insigne E prćclarum, vereque coelestis harmonić, Authore nunquam satis lau= | dato Musico, Henrico Isaac, Diui quondam Cósaris Maximiliani Symphonista Regio, opus | inquam, illustirs Isaci, officina dignum, E propter compositionis artistium, Ẽ cygneam | venusatatem, adeo vt ex sćcundissimo tanti artificis pectore, vere | emanasse videatur. | Nornbergć imprimebat Hieronymus Formschneider | Cum gratia priuilegio Cósareć Maiestatis ad quinquennium. I Anno 1550. I [[S:] DISCANTVS | DOMINICARVM A TRINITATE | VSQVE AD ADVENTUM | DOMINI. [A:] ALTVS | DOMINICARVM A TRINITATE | VSQVE AD ADVENTUM DOMINI. [B:] BASSVS | DOMINICARVM A TRINITATE | VSQVE AD ADVENTUM | DOMINI.]. 1550, Hieronymus Formschneider Nürnberg, RISM I 89, II 89.

4. Antiphonae variae scriptae 9 Theil in 4 to

5. Responsoria de tempore 2 Theil in 4 to

6. Canticum B[eatae] V[irginis] M[ariae] Christoph[ori] Demantij 4 Theil in 4to

Christoph Demantius (1567-1643): Probably Trias precum vespertinarum, qua continentur: canticum B. Mariae Virginis, intonationes cum psalmis, et clausulae in precibus vespertinis consuetae quas Benedicamus vocant; et ad octo usitatos tonos: $\mathcal{E}$ ad duodecim modos musicos, tam quaternis, quam quinis $\mathcal{E}$ senis vocibus: eleganti harmonia, $\mathcal{E}$ quibusvis instrumentorum musicorum generibus communi, expressa et decantata. 1602 Nürnberg, Conrad Agricola (Katharina Dietrich) RISM D 1533.

7. Iten Canticum Auth[ore] Wussin 1 Theil in 4to unidentified

8. Missae Joan[nes] Stadlmajer 5 Theil in 4 to Johann Stadlmayr (c1575-1648): considering the number of vocals it could probably be following masses: Missae breves a IV. cum una pro defunctis et alia V. voc. concertatae. 1641 Innsbruck, Michael Wagner RISM S 4299 a Missae breves a IV. cum una pro defunctis et alia V. voc. concertatae...editio secunda. 1660 Innsbruck, Michael Wagner RISM S 4300.

9. Variae Antiph[onae] Theodor[us] Ring 3 Theil in 4to unidentified

10. Thesaurus Musicae Orlandi de Lasso 6 Theil in quarto

Orlando di Lasso (c1532-1594): the work is impossible to identify due to the amount of sources.

11. Responsoria B[eatae] V[irginis] M[ariae] Petri Joannelli 3 Theil in 4to

Pietro Giovanelli (date of birth and death unknown): impossible to identify precisely.

12. Psalmi Davidis germanice 4 Theil in 4 to 
unidentified

13. Responsoria de tempore 3 Theil in 4to

unidentified

14. Centuria Concertuum Ludovi[ci] Diadana (!) 4 Theil in 4to

Lodovico Viadana (c1560-1627): the title Centuria Concertuum points towards the collection Cento Concerti ecclesiastici from 1602. Cento concerti ecclesiastici, a una, a due, a tre, Ev a quattro voci, con il basso continuo per sonar nell'organo, nova inventione commoda per ogni sorte de cantori, Ẽ per gli organisti ... opera duodecima ([jeweils mit Zwischentitel:] Concerti a due voci, Concerti a tre voci ...). 1602 Venezia, Giacomo Vincenti RISM V 1360.

15. Cantiones Jacobi Händl 9 Theil in 4to

Iacobus Gallus (1550-1591): Sacrae cantiones de praecipuis festis per totum annum, quae ex sancto ecclesiae catholicae usu ita sunt ordinatae et dispositae, ut omni tempore inservire queant, quatuor, quinque, sex, octo et plurium vocum. 1597 Nürnberg, Alexander Theodoricus RISM H 1990.

16. Symphoniae Sacrae Joan[nis] (!) Ertelij 7 Theil in 4to

Sebastian Ertel (c1550-1618): the same title bears the collection Symphoniae sacrae, ad Dei divorumque laudes in ecclesia qua instrumentis, qua vivis hominum vocibus decantandas accommodatae, quibus sexta vox initium, decima vero finem dabit. 1611. The number of vocals, however, does not match.

17. Vesperae Scriptae 5 Theil in 4 to unidentified

18. Psalmi Wilhelmi Nortwinck (!) 4 Theil in 4 to

Wilhelm Nordwig (unknown): in the typographic workshop of Johann vom Berg and Ulrich Neuber was published in 1553 Tomus secundus Psalmorum selectorum, quator et plurium vocum. It was published in several volumes, however, Nordwig is mentioned only in the second volume. ${ }^{33}$

19. Selecti moduli Jacob[i] de Gerle 4 Theil in 4to

Jacobus de Kerle (c1531-1591): Selectiorum aliquot modulorum, qui in sacris templis ... partim quatuor, partim quinque et octo vocibus, decantari solent. 1585 Prag, Georg Nigrinus RISM K 455.

20. Officia Missarum Franc[isci] Sale 3

Franz Sales (c1540-1599): Officiorum Missalium, quibus Introitus, Alleluia et Communiones de omnibus omnium sanctorum, per totum anni circulum, diebus festis $\mathcal{E}$ solennibus quinque $\mathcal{E}$ sex vocum continentur, liber secundus. 1594 Praha, Georg Nigrinus RISM S 395, SS 395. Officiorum Missalium, quibus Introitus, Alleluia et Communiones de omnibus omnium sanctorum, per totum anni circulum, diebus festis $\mathcal{E}$ solennibus quinque E Sex vocum continentur, liber tertius E ultimus. 1596 Praha, Georg Nigrinus RISM S 397.

21. Theil in 4 to

unidentified

22. Cantiones Jacob[i] Regnardi 2 Theil in 4to

Jacob Regnart (c1540-1599), impossible to identify precisely.

23. Thesaurus Musicus Joan[nis] Montani 2 Theil in 4to

Perhaps Thesaurus musicus continens selectissimas octo, septem, sex, quinque et quatuor vocum harmonias, tam a veteribus quam recentioribus symphonistis compositas et ad omnis

33 DANĚK, Petr. Historické tisky, op. cit., p. 86. 
generis instrumenta musica accomodatas (...) Tomi primi continentis cantiones octo vocum (...) Nürnberg, J. Montanus et U. Neuber, 1564 (already not complete in the time when the inventory was written)

24. Mariale pro festis B[eatae] V[irginis] M[ariae] Jacob[i] Regn[ardi] 2 Theil in 4to

Jacob Regnart: Mariale, hoc est: Opusculum sacrarum cantionum pro omnibus Beatissimae Virginis Mariae festivitatibus, cum quatuor, quinq; sex E octo vocibus, nunquam antea in lucem editum. 1588 Innsbruck, Johannes Paur RISM 1588.

25. Cantiones Orlandi Lassi (!) 2 Theil in 4to

Orlando di Lasso, impossible to identify precisely.

26. Antiphonale 1 Theil in folio

27. Officia Missarum Franc[isci] Sale 1 Theil in folio

Franz Sales (c1540-1599): probably Patrocinium musices. Missarum solenniorum, tam sanctorum quam festorum officia labentis anni, in catholicae ecclesiae usum, harmonice contra punctum ac suavissime concinnata ... primus tomus. 1589 München, Adam Berg RISM S 392.

28. Psalterium Davidis Typo gottica 1 Theil in folio

29. Magnificat 4 vocum ejusdem Auth[ore] 1 Theil in folio

30. Missae Orlandi Lassi (!) 1 Theil in folio

Orlando di Lasso: impossible to identify precisely.

31. Antiphonale majus gottice scriptum 1 Theil.

Nebst diessen gebundenen befinden sich Viel anders ungebundenes Musicalia so mehresten theils geschrieben, verwirret und untereinander gestreuet seyn.

Of the 31 entries in the inventory it was possible to identify 13 authors and 11 compositions. Those compositions refine the repertoire of the deanery church. With the mentioned sheet music a question of their performance arises. The compositions come mostly from the era of High Renaissance or Early Baroque. At the time of writing the inventory the repertoire was more than 150 years old. That could mean that the compositions perhaps did not belong to regular production, however, they were precious enough for the church to thoroughly preserve them.

\section{Conclusion}

Deeper analysis of the inventories from Most is relatively thorough probe to musical practice of those times. The Corporis Christi Confraternity participated in the development of musical production and events as well as during regular church ceremonies. Regarding the instrumentations of the deanery church it is evident that the church also provided quality conditions for interpretative practice. The study then focused mostly on the inventory from 1761 which includes a detailed list of sheet music. The list provides information about the liturgical purpose and authorship of mentioned compositions. Most still represents a location whose musical history will be further studied. 


\section{Bibliography}

CORI, Johann Nepomuk, Ferdinand von HOCHSTETTER a Franz SIEGEL. Geschichte der königl. Stadt Brüx bis zum Jahre 1788. Brüx: Im Verlage der Stadt Brüx, 1889.

ČERNÝ, Milan. Chrámoví hudebníci a hudebně liturgická praxe při duchcovském kostele zvěstování Panny Marie v první polovině 18. století. In Porta Bohemica: sbornik historických prací, č. 8. Ústí nad Labem: Albis international, 2001.

DANĚK, Petr. Historické tisky vokálni polyfonie, rané monodie, hudebni teorie a instrumentálni hudby v českých zemich do roku 1630: se soupisem tisků z let 1488-1628 uložených v Čechách. Praha: KLP, 2015.

EITNER, Robert. Biographisch-Bibliographisches Quellen-Lexikon der Musiker und Musikgelehrten der christlichen Zeitrechnung bis zur Mitte des neunzehnten Jahrhunderts. Leipzig: Breitkopf und Härtel, 1900-1904.

FÖRSTER Jürgen. Stavitel Jacob Haylmann ze Schweinfurtu, město Annaberg a jejich vliv na vybudování děkanského chrámu v Mostě. In 500 let kostela Nanebevzetí Panny Marie v Mostě. V Mostě: Oblastní muzeum, 2018.

KONRÁD, Karel. Dějiny posvátného zpě̃u staročeského. Díl 2., č. 1., od XV. věku do zrušení literátskych bratrstev, Věk a dějiny literárních bratrstev. Praha: Cyrillo-Methodějská kněhtiskárna, 1883.

MANNLOVÁ, Heide. Kulturni památka Most: Děkanský kostel a jeho stavitelé. Praha: Pro odbor kultury a Okresní muzeum v Mostě k tisku připravila Propagační tvorba, 1989.

MYŠIČKA, Martin. Kniha účtů ke sbírce na stavbu děkanského kostela. In 500 let kostela Nanebevzeti Panny Marie v Mostě. V Mostě: Oblastní muzeum, 2018.

PÁTKOVÁ, Hana. Bratrstvie ke cti Božie: poznámky ke kultovni činnosti bratrstev a cechů ve středoveékých Čechách. Praha: KLP, 2000.

VLČEK, Pavel, Dušan FOLTÝN a Petr SOMMER. Encyklopedie českých klášterů. Praha: Libri, 1997.

VOIT, Petr. Encyklopedie knihy: starši knihtisk a př́buzné obory mezi polovinou 15. a počátkem 19. století: papir, pismo a pismolijectví, knihtisk a jiné grafické techniky, tiskaři, nakladatelé, knihkupci, ilustrátoři a kartografové, literárni typologie, textové a výtvarné prvky knihy, knižni vazba, knižni obchod. 2. vyd. Praha: Libri ve spolupráci s Královskou kanonií premonstrátů na Strahově, 2008.

\section{Sources}

State Archiv Prague, Archive of the Prague Archdioecesis (APA), box nr. 2395.

State regional archive Most, Archive of the City of Most (1315-1945), Inv. nr. 3438 (auxiliary musicians in the deanery church 1677-1803).

State regional archive Most, Archive of the City of Most (1315-1945), Inv. nr. 2752 (inventories of the deanery church).

State regional archive Most, Archive of the City of Most (1315-1945), Inv. nr. 2913 (contract between the town of Most and the organ builder David Decker from Zeilenrode in Voigtland).

State regional archive Most, Archive of the City of Most (1315-1945), Inv. nr. 2915 (contract between the town of Most and the organ builder Wenzel Starcke from Loket / Ellbogen). 
